\title{
Equidistribution of Matrix-Power Residues Modulo One
}

\author{
By Joel N. Franklin
}

1. Introduction. In numerical analysis artificial random numbers are generated by recurrence formulas of the type

$$
x_{n+1}=\left\{N x_{n}+\theta\right\} \quad(n=0,1,2, \cdots) .
$$

Here $\{y\}=y-[y]=$ the fractional part of $y$. The number $N$ is an integer $>1$. The number $x_{0}$ is a given initial value such that $0 \leqq x_{0}<1$. The number $\theta$ is fixed. Some early references to numerical work with sequences of the type (1) are given by 0 . Taussky and J. Todd in [1]. Regarding the sequence $x_{n}$ as a function of $x_{0}$, I proved in [2] that for almost all $x_{0}$ the sequence $x_{n}$ is equidistributed modulo 1 , i.e.,

$$
\lim _{k \rightarrow \infty} \frac{1}{k} \sum_{a \leqq x_{n}<b ; n=0, \cdots, k-1} 1=b-a
$$

whenever $0 \leqq a<b \leqq 1$.

The purpose of this paper is to generalize the preceding result to vector-matrix recurrence formulas

$$
x^{(n+1)}=\left\{A x^{(n)}+b\right\} \quad(n=0,1, \cdots) .
$$

Here each $x^{(n)}$ is a $d$-dimensional column vector, $b$ is a $d$-dimensional column vector, and $A$ is a $d \times d$ matrix with integer components. In the preceding case (1), $d=1$, $A=N$, and $b=\theta$. By $\{y\}$ for a vector $y$ with real components $y_{i}$ is meant the vector with components $\left\{y_{i}\right\}$. The vector $x^{0}$-with parentheses removed around the superscript-is given in the unit cube $C_{d}$ of $d$ dimensions,

$$
C_{d}: 0 \leqq x_{i}<1 \quad(i=1, \cdots, d) .
$$

All the vectors $x^{n}$ lie in $C_{d}$. The main result of the paper is: $A$ sufficient condition that $x^{n}$ be equidistributed for almost all $x^{0}$ is that the matrix $A$ be nonsingular and have no eigenvalue which is a root of unity; if $b=0$, so that $x^{n+1}=\left\{A x^{n}\right\}$, the condition is necessary as well as sufficient.

This result has applications to numerical analysis and to the theory of numbers. In [3] the one-dimensional sequences (1) were analyzed at length. It was shown there that for $d>1$ the successive $d$-tuples

$$
\left(x_{0}, \cdots, x_{d-1}\right), \quad\left(x_{d}, \cdots, x_{2 d-1}\right), \quad\left(x_{2 d}, \cdots, x_{3 d-1}\right), \quad \cdots
$$

cannot be equidistributed in $C_{d}$. In other words, the proportion of these vectors, taken sequentially, which lie in a subregion $R$ of $C_{d}$ cannot generally be expected to approach the ratio (volume of $R$ )/(volume of $C_{d}$ ) = volume of $R$. However, as the result stated in the last paragraph shows, if $A=\operatorname{diag}(N, N, \cdots, N)$, where $N=$ integer $>1$, the vectors defined by (3) are equidistributed for almost all

Received December 5, 1963. 
choices of the $d$ components of the initial vector $x^{0}$. For example, if $d=3$ and $b=0$, we find that the vectors $x^{n}=\left(u_{n}, v_{n}, w_{n}\right)(n=0,1, \cdots)$ defined by

$$
u_{n+1}=\left\{N u_{n}\right\}, \quad v_{n}=\left\{N v_{n+1}\right\}, \quad w_{n}=\left\{N w_{n+1}\right\}
$$

are equidistributed in the unit cube $C_{3}$ for almost all initial values $u_{0}, v_{0}, w_{0}$.

In the theory of numbers we obtain the following sort of result: For almost all real initial values $f_{0}, f_{1}$, the Fibonacci sequence defined by

$$
f_{n+1}=f_{n}+f_{n-1} \quad(n=1,2, \cdots)
$$

is equidistributed by twos modulo one, i.e.,

$$
\lim _{k \rightarrow \infty} \frac{1}{k} \sum_{a_{1} \leqq f_{n}<b_{1} ; a_{2} \leqq f_{n+1}<b_{2} ; n=0, \cdots, k-1} 1=\left(b_{1}-a_{1}\right)\left(b_{2}-a_{2}\right)
$$

whenever $0 \leqq a_{1}<b_{1} \leqq 1$ and $0 \leqq a_{2}<b_{2} \leqq 1$. Setting $a_{2}=0, b_{2}=1$, we obtain the weaker result that almost all Fibonacci sequences are equidistributed modulo one.

2. The Theorems of Weyl and Riesz. A sequence of $d$-dimensional, real vectors

$$
x^{(n)}=\left(x_{1}{ }^{n}, x_{2}{ }^{n}, \cdots, x_{d}{ }^{n}\right) \quad(n=0,1, \cdots)
$$

is said to be equidistributed modulo one if

$$
\lim _{k \rightarrow \infty} \frac{1}{k} \sum_{a_{i} \leqq\left\{x_{i}{ }^{n}\right\}<b_{i}} \sum_{(i=1, \cdots, d) ; n=0, \cdots, k-1} 1=\prod_{i=1}^{d}\left(b_{i}-a_{i}\right)
$$

whenever $0 \leqq a_{i}<b_{i} \leqq 1(i=1, \cdots, d)$. We shall use the following theorem of H. Weyl [4]:

Theorem. A sequence (1) of d-dimensional vectors $x^{(n)}$ is equidistributed modulo one if and only if

$$
\lim _{k \rightarrow \infty} \frac{1}{k} \sum_{n=0}^{k-1} \exp 2 \pi i\left(j_{1} x_{1}{ }^{n}+j_{2} x_{2}{ }^{n}+\cdots+j_{d} x_{d}{ }^{n}\right)=0
$$

for all combinations of integers $j_{1}, \cdots, j_{d}$ except $j_{1}=\cdots=j_{d}=0$.

We shall also need the ergodic theorem of F. Riesz; see [5] and [2]:

ThEOREM. Let a measurable set $\Omega$ be given, of finite or infinite measure, the corresponding measure and integral being defined according to Lebesgue, or more generally, by means of a distribution of positive masses. That being the case, let us designate by $T$ a point-transformation which is single-valued (but not necessarily one-to-one) from $\Omega$ onto itself; and let us suppose that $T$ conserves measure in the sense that, $E$ being $a$ measurable set, TE its transform, and $E^{\prime}$ the set of points $P$ whose images appear in $T E$, the sets $E^{\prime}$ and $T E$ have the same measure. Then, if $f_{1}(P)$ is an integrable function and $f_{k}(P)=f_{1}\left(T^{k-1} P\right)$, the arithmetic mean of the functions $f_{1}, f_{2}, \cdots, f_{n}$ converges almost everywhere, as $n \rightarrow \infty$, to an integrable function $\phi(P)$ which is invariant (almost everywhere) under T. If $\Omega$ is of finite measure,

$$
\int_{\Omega} \phi(P)=\int_{\Omega} f_{1}(P)
$$


3. Measure-Preserving Congruences Modulo One. Let $A$ be a $d \times d$ matrix with real components, and let $b$ be a $d$-component column vector. We define a transformation $y=T x$ of the $d$-dimensional unit cube $C_{d}$ into itself by the congruence

$$
y \equiv A x+b(\bmod 1)
$$

by which we mean $y=\{A x+b\}$ or, equivalently,

$$
y_{i} \equiv \sum_{j=1}^{d} a_{i j} x_{j}+b_{i}(\bmod 1) \quad(i=1, \cdots, d) .
$$

We wish to determine when this transformation is measure-preserving.

First we remark that the congruence (1) is measure-preserving if and only if the congruence

$$
w \equiv A x(\bmod 1)
$$

is measure-preserving. That is because the congruence (1) may be composed of two transformations, $w=\{A x\}$ and $y=\{w+b\}$. Since the second transformation is one-to-one and measure-preserving, the composite transformation (1) is measure-preserving if and only if the first transformation (2) is measure-preserving.

Second, we remark that the transformation $T$ is measure-preserving if and only if

$$
\int_{C_{d}} f(P)=\int_{C_{d}} f(T P)
$$

for all scalar functions $f$ which are measurable in $C_{d}$. This elementary remark is justified by Riesz in [5].

Lemma. Let $K$ be the set of nonzero d-dimensional column-vectors $k$ with integer components. Let $K_{1}$ be the set of d-dimensional real column vectors with at least one component equal to a nonzero integer. Then the congruence $y \equiv A x+b(\bmod 1)$ is measure-preserving in $C_{d}$ if and only if the transpose matrix $A^{*}$ maps $K$ into $K_{1}$.

Proof. Let the measurable function $f(P)=f(x)$ have the Fourier series

$$
f(x) \sim c(0)+\sum_{k \in K} c(k) \exp 2 \pi i k^{*} x .
$$

Since the Fourier series is multiply periodic, the congruence $T$ is measure-preserving if and only if

$$
c(0)=\int_{C_{d}} f(x) d x=\int_{C_{d}} f(A x) d x
$$

for all measurable $f$. But

$$
\begin{aligned}
\int_{C_{d}} f(A x) d x & =c(0)+\sum_{k \in \boldsymbol{K}} c(k) \int_{C_{d}} \exp 2 \pi i k^{*} A x d x \\
& =c(0)+\sum_{k \in \boldsymbol{K}} c(k) \int_{C_{d}} \exp 2 \pi i\left(A^{*} k\right)^{*} x d x
\end{aligned}
$$

Therefore, $T$ is measure-preserving if and only if 


$$
\int_{C_{d}} \exp 2 \pi i\left(A^{*} k\right)^{*} x d x=0 \quad \text { for all } \quad k \in K
$$

which is true if and only if $A^{*} k \in K_{1}$ for all $k \in K$.

The lemma shows that, if $d=1$, the congruence $y \equiv A x+b$ is measure-preserving if and only if $A$ is a nonzero integer. However, if $d>1$, the matrix $A$ may have noninteger coefficients. For example, the congruence

$$
\left(\begin{array}{l}
y_{1} \\
y_{2}
\end{array}\right) \equiv\left(\begin{array}{rr}
0 & -6 \\
\frac{1}{2} & 1
\end{array}\right)\left(\begin{array}{l}
x_{1} \\
x_{2}
\end{array}\right)+\left(\begin{array}{l}
b_{1} \\
b_{2}
\end{array}\right)(\bmod 1)
$$

is measure-preserving. To see this, we observe that

$$
A^{*} k=\left(\begin{array}{c}
\frac{1}{2} k_{2} \\
-6 k_{1}+k_{2}
\end{array}\right) \text {. }
$$

If $k \in K$, the first component $k_{2} / 2$ is a nonzero integer unless $k_{2}$ is zero or odd. If $k_{2}=0$, the second component $=-6 k_{1}=$ integer $\neq 0$; if $k_{2}$ is odd, $-6 k_{1}+k_{2}=$ even integer + odd integer $\neq 0$. Therefore, $A^{*}$ maps $K$ into $K_{1}$.

In the rest of the paper we shall suppose that $A$ has all components equal to integers.

Theorem. If all the components of $A$ are integers, the congruence $y \equiv A x+b$ (mod 1) is measure-preserving if and only if $\operatorname{det} A \neq 0$.

Proof. This result follows immediately from the lemma. Since $A$ has integer components, if $\operatorname{det} A=0$ there is a vector $k \in K$ such that $A^{*} k=0$, which is not in $K_{1}$. If $\operatorname{det} A \neq 0$, all vectors $A^{*} k$ are nonzero vectors with integer components when $k \in K$, so that $A^{*} k \in K \in K_{1}$.

4. Ergodic Congruences Modulo One. We shall say that a measure-preserving transformation $y=T x$ from the $d$-dimensional unit cube into itself is ergodic if the only measurable functions $\phi(x)$ for which

$$
\phi(x)=\phi(T x) \quad \text { almost everywhere in } C_{d}
$$

are the functions $\phi(x)=$ constant a.e. (almost everywhere).

Lemma. Let $B$ be a $d \times d$ matrix with integer components. Let $K$ be the set of nonzero d-dimensional column-vectors with integer components. Then the sequence of vectors $k, B k, B^{2} k, \cdots$ is unbounded for every $k$ in $K$ if and only if $B$ has no eigenvalue which is zero or a root of unity.

Proof. Suppose that for some $k$ in $K$ the sequence $B^{j} k$ is bounded. Since $B$ and $k$ have integer components, each of the vectors $B^{j} k$ must be one of the finite number of integer-component vectors which lie in some bounded subset of $d$-dimensional Euclidean space. Therefore, $B^{r} k=B^{s} k$ for some $r>s$. If $B$ has no zero eigenvalue, $B$ is nonsingular and $B^{q} k=k$ for $q=r-s$. But then

$$
0=\operatorname{det}\left(B^{q}-I\right)=\prod_{j=0}^{q-1} \operatorname{det}\left(B-\omega^{j} I\right)
$$

where $\omega=\exp (2 \pi i / q)$. Then one of the roots of unity $\omega^{j}$ is an eigenvalue of $B$.

Conversely, if $B$ has a zero eigenvalue, since $B$ has integer components, there 
is an eigenvector $k$ in $K$ such that $0=B k=B^{2} k=\cdots$, a bounded sequence. If $B$ has an eigenvalue which is a $q$ th root of unity, then $B^{q}$ has 1 as an eigenvalue. Then there is an eigenvector $k$ in $K$ such that $B^{q} k=k$, and the sequence $B^{j} k$ is periodic, hence bounded.

Theorem. Let $A$ be a nonsingular $d \times d$ matrix with integer components, and let $b$ be a d-dimensional column-vector with real components. Then the measure-preserving congruence $y \equiv A x+b(\bmod 1)$ is ergodic if $A$ has no eigenvalue which is a root of unity. The congruence $y \equiv A x(\bmod 1)$ is ergodic if and only if $A$ has no eigenvalue which is a root of unity.

Proof. Let $T x \equiv A x+b(\bmod 1)$, where $b$ is a vector with real components, and $A$ is a nonsingular matrix with integer components and with no eigenvalue equal to a root of unity. Then $B=$ transpose of $A=A^{*}$ has no eigenvalue which is zero or a root of unity. According to the lemma, $B^{j} k$ is unbounded as $j \rightarrow \infty$ for every $k$ in $K$. ing,

Let $\phi(x)$ be any measurable function satisfying (1). Since $T$ is measure-preserv-

$$
\phi(x)=\phi\left(T^{j} x\right) \text { a.e. for all } j=1,2, \cdots .
$$

The measurable function $\phi(x)$ has a Fourier series

$$
\phi(x) \sim a(0)+\sum_{k \in \mathbb{K}} a(k) \exp 2 \pi i k^{*} x .
$$

Furthermore,

$$
T^{j} x \equiv A^{j} x+b^{(j)}(\bmod 1)
$$

where $b^{(j)}=b+A b+\cdots+A^{j-1} b$. Therefore,

$$
\phi\left(T^{j} x\right) \sim a(0)+\sum_{k \in \mathbb{K}} a(k) \exp 2 \pi i k^{*}\left(A^{j} x+b^{(j)}\right)
$$

or, equivalently, with $B=A^{*}$,

$$
\phi\left(T^{j} x\right) \sim a(0)+\sum_{k \in \mathbb{K}}\left(a(k) \exp 2 \pi i k^{*} b^{(j)}\right) \exp 2 \pi i\left(B^{j} k\right)^{*} x
$$

Therefore,

$$
\begin{aligned}
a(k) \exp 2 \pi i k^{*} b^{(j)} & =\int_{C_{d}} \phi\left(T^{j} x\right) \exp \left(-2 \pi i\left(B^{j} k\right)^{*} x\right) d x \\
& =\int_{C_{d}} \phi(x) \exp \left(-2 \pi i\left(B^{j} k\right)^{*} x\right) d x
\end{aligned}
$$

Since $B^{j} k$ is unbounded for each $k$ in $K$, the integrals (6) tend to zero for some subsequence of $j$ tending to $\infty$. But the left-hand side of (6) has modulus $|a(k)|$ for all $j$. Therefore, $a(k)=0$ for all $k \in K$. Then the Fourier series for $\phi(x)$ consists only of the constant term $a(0)$. Therefore, $\phi(x)$ equals this constant almost everywhere.

If $T x \equiv A x(\bmod 1)$, i.e., if $b=0$, we can show that the transformation is ergodic only if $A$ has no eigenvalue which is a root of unity. Suppose that $A$, and therefore $B$, have eigenvalues which are $q$ th roots of unity. Then $B^{q} k=k$ for some 
$k$ in $K$. Let $p$ be the smallest positive integer such that $B^{p} k=k$. Since $A$, and therefore $B$, is nonsingular, no two of the vectors, $k, B k, \cdots, B^{p-1} k$ are equal. Therefore, the function

$$
\phi(x)=\sum_{j=0}^{p-1} \exp \left(2 \pi i k^{*} A^{j} x\right)
$$

is nonconstant. But $\phi(x)=\phi(T x)$, since $k^{*} A^{p}=\left(B^{p} k\right)^{*}=k^{*}$. Therefore, $T$ is not ergodic. This completes the proof of the theorem.

If $b \neq 0$, the transformation $T x \equiv A x+b(\bmod 1)$ may be ergodic even if $A$ has an eigenvalue which is a root of unity. For example, the transformation $T x \equiv$ $x+b$ is ergodic if and only if the components of $b$ are rationally independent, i.e., if $k^{*} b \neq$ integer for all $k$ in $K$. This result follows immediately from the uniqueness of the Fourier series of a measurable function $\phi(x)$.

A more interesting question arises when $A \neq I$. For example, consider the transformation

$$
T x \equiv\left(\begin{array}{ll}
2 & 0 \\
0 & 1
\end{array}\right)\left(\begin{array}{l}
x_{1} \\
x_{2}
\end{array}\right)+\left(\begin{array}{c}
0 \\
\sqrt{2}
\end{array}\right)(\bmod 1)
$$

If $\phi(x)$ has the Fourier series (3), then

$$
\phi\left(T^{j} x\right) \sim a(0)+\sum_{k \in K} a_{j}(k) \exp 2 \pi i\left(2^{j} k_{1} x_{1}+k_{2} x_{2}\right)
$$

where $a_{j}(k)=a(k) \exp 2 \pi i k_{2} \sqrt{ } \overline{2}$. Then the invariance (1) implies

$$
a_{j}(k)=\int_{0}^{1} \int_{0}^{1} \phi(x) \exp -2 \pi i\left(2^{j} k_{1} x_{1}+k_{2} x_{2}\right) d x_{1} d x_{2} .
$$

Letting $j \rightarrow \infty$, we see that $a(k)=0$ unless $k_{1}=0$. But then

$$
\phi\left(x_{1}, x_{2}\right) \sim \sum_{k_{2} \neq 0}^{\infty} a\left(0, k_{2}\right) \exp 2 \pi i k_{2} x_{2} .
$$

Now the irrationality of $\sqrt{2}$ implies that $a\left(0, k_{2}\right)=0$ for all $k_{2} \neq 0$. Therefore, the transformation (8) is ergodic.

ThEOREM. Let

$$
\begin{aligned}
& y_{1} \equiv N x_{1}+b_{1}(\bmod 1) \\
& y_{s} \equiv x_{s}+b_{s} \quad(s=2, \cdots, d)
\end{aligned}
$$

where $N$ is an integer with absolute value $>1$, and the $b_{s}$ are real. This measurepreserving transformation is ergodic if and only if $k_{2} b_{2}+\cdots+k_{d} b_{d} \neq$ integer for any integers $k_{2}, \cdots, k_{d}$ which are not all zero.

Proof. This theorem is an immediate and obvious generalization of the preceding example.

\section{Equidistribution of Matrix-Power Residues.}

Theorem. Let $A$ be a $d \times d$ matrix with integer components. Let $b$ be a d-dimensional column vector with real components. Given the vector $x=x^{(0)}$, construct the sequence $x^{(j)}$ by the recurrence formula 


$$
x^{(j+1)} \equiv A x^{(j)}+b(\bmod 1)
$$

for $j=0,1, \cdots$. This sequence is equidistributed modulo one for almost all $x$ if $A$ has no eigenvalue equal to zero or a root of unity; if $b=0$, the sequence is equidistributed for almost all $x$ if and only if $A$ has no eigenvalue equal to zero or a root of unity.

Proof. If $A$ has no eigenvalue equal to zero, $A$ is nonsingular; and, according to the theorem in Section 3 , the transformation $T x \equiv A x+b(\bmod 1)$ is measurepreserving. Therefore, by the Riesz ergodic theorem, for all measurable functions $f$

$$
\frac{1}{k} \sum_{j=0}^{k-1} f\left(x^{(j)}\right) \rightarrow \phi(x) \quad \text { as } \quad k \rightarrow \infty
$$

for almost all $x=x^{(0)}$, where $\phi(x)=\phi(T x)$ a.e. By the first theorem in Section 4 , if $A$ is nonsingular and has no eigenvalue which is a root of unity, $\phi(x)=$ constant a.e. By the Riesz ergodic theorem, since the $d$-dimensional unit cube $C_{d}$ has finite measure $=1$, the constant $\phi$ has the integral

$$
\int_{C_{d}} f(x) d x=\int_{C_{d}} \phi d x=\phi .
$$

If $0 \leqq a_{i}<b_{i} \leqq 1(i=1, \cdots, d)$ define

$$
\begin{aligned}
& f(x)=f\left(x_{1}, \cdots, x_{d}\right)=1 \quad \text { for } a_{i} \leqq x_{i}<b_{i} \quad(i=1, \cdots, d) \\
& =0 \quad \text { elsewhere in } C_{d} \text {. }
\end{aligned}
$$

From (2) and (3) we have the result, for almost all $x$, that the sequence $x^{(j)}$ is equidistributed in $C_{d}$.

For $b=0$ we must prove the "only if" part of the theorem. First suppose that $A$ has an eigenvalue equal to zero. Then $A^{*} k=0$ for some $k$ in $K$. Let

$$
f(x)=\exp 2 \pi i k^{*} x .
$$

Since $f(x)$ is Riemann-integrable, we must have

$$
\lim _{n \rightarrow \infty} \frac{1}{n} \sum_{j=0}^{n-1} f\left(x^{(j)}\right)=\int_{C_{d}} f(x) d x
$$

if $x^{(j)}$ is equidistributed; for a proof of this result see Koksma [6]. From (5) we have

$$
f\left(x^{(j)}\right)=\exp 2 \pi i k^{*} A^{j} x=1 \quad(j \geqq 1) .
$$

Therefore, the limit on the left-hand side of (6) equals one. Since the integral of $f(x)$ equals zero, equation (6) is false; and the sequence $x^{(j)}$ cannot be equidistributed.

Finally, for $b=0$ suppose that $A$ is nonsingular but that $A$ has an eigenvalue which is a root of unity. Construct the nonconstant, Riemann-integrable function $\phi(x)$ defined in formula $(7)$ of Section 4 . Since $\phi(x)=\phi(T x)$, we have

$$
\frac{1}{n} \sum_{j=0}^{n-1} \phi\left(x^{(j)}\right)=\phi\left(x^{(0)}\right)=\phi(x) \quad \text { for all } n .
$$

But

$$
\int_{C_{d}} \phi(x) d x=0
$$


Therefore, the sequence $x^{(j)}$ cannot be equidistributed. This completes the proof of the theorem.

6. Application to Numerical Analysis. In Monte Carlo calculations in $d$ dimensions, the basic property required of pseudo-random vectors $x^{(j)}$ is usually the property (6) of Section 5. This property is equivalent to the equidistribution of the $x^{(j)}$. The reader is now referred back to the next to the last paragraph of Section 1.

7. Equidistribution of Fibonacci Sequences. We shall say that a sequence of real numbers $x_{n}$ is equidistributed by d's modulo one if the sequence of successive $d$-tuples

$$
x^{(n)}=\left(\begin{array}{c}
x_{n+1} \\
x_{n+2} \\
\vdots \\
x_{n+d}
\end{array}\right) \quad(n=0,1, \cdots)
$$

is equidistributed modulo one, as defined in Section 2. This concept was considered at length in [3]. For $d=1$ we have the usual definition for the equidistribution of $x_{n}$ modulo one. A sequence equidistributed by $d$ 's for $d>1$ is equidistributed by $r$ 's for $1 \leqq r<d$, but the converse is false.

Theorem. Let a general Fibonacci sequence $x_{n}$ be defined by

$$
x_{n}=a_{1} x_{n-1}+a_{2} x_{n-2}+\cdots+a_{d} x_{n-d} \quad(n>d)
$$

where $a_{1}, a_{2}, \cdots, a_{d}$ are integers. Then for almost all real initial values $x_{1}, \cdots, x_{d}$ the sequence $x_{n}$ is equidistributed by d's modulo one if and only if

$$
z^{d} \neq a_{1} z^{d-1}+a_{2} z^{d-2}+\cdots+a_{d}
$$

for $z=0$ or for $z=$ a root of unity.

Proof. Define the matrix

$$
A=\left(\begin{array}{ccccc}
0 & 1 & 0 & \cdots & 0 \\
0 & 0 & 1 & \cdots & 0 \\
\cdot & \cdot & \cdot & \cdots & \cdot \\
0 & 0 & 0 & \cdots & 1 \\
a_{d} & a_{d-1} & a_{d-2} & \cdots & a_{1}
\end{array}\right) .
$$

The relation (2) is equivalent to the vector-matrix relation

$$
x^{(n+1)}=A x^{(n)} \quad(n=0,1, \cdots) .
$$

The eigenvalues of $A$ are the roots of the equation

$$
0=\operatorname{det}(z I-A)=z^{d}-a_{1} z^{d-1}-\cdots-a_{d} .
$$

The theorem now follows directly from the result in Section 5 .

California Institute of Technology

Pasadena, California 
1. O. TAUSSKY \& J. TODD, "Generation of pseudo-random numbers," Symposium on Monte Carlo Methods, H. A. Meyer, Editor, John Wiley and Sons, New York, 1956, p. 15-18.

2. J. N. FRANKLIN, "On the equidistribution of pseudo-random numbers," Quart. Appl. Math., v. 16, 1958, p. 183-188.

3. J. N. FrANKLIN, "Deterministic simulation of random processes," Math. Comp., v. 17,1963, p. $28-59$.

4. H. WEYL, "Über die Gleichverteilung von Zahlen modulo Eins," Math Ann., v. 77, 1916, p. 313-352.

5. F. RIEsz, "Sur la théorie ergodique," Comment. Math. Helv., v. 17, 1945, p. 221.

6. J. F. Koкsma, Diophantische Approximationen, Chelsea, New York, 1936. 